\title{
Infant nutrition in relation to eating behaviour and fruit and vegetable intake at age 5 years
}

\author{
Lisanne M. Möller ${ }^{1}$, Marieke L. A. de $\operatorname{Hoog}^{1,2 *}$, Manon van Eijsden ${ }^{2,3}$, Reinoud J. B. J. Gemke ${ }^{4}$ and \\ Tanja G. M. Vrijkotte ${ }^{1}$ \\ ${ }^{1}$ Department of Public Health, Academic Medical Centre, University of Amsterdam, PO Box 22660, 1100 DD Amsterdam, \\ The Netherlands \\ ${ }^{2}$ Department of Epidemiology, Documentation and Health Promotion, Public Health Service, PO Box 2200, \\ 1000 CE, Amsterdam, The Netherlands \\ ${ }^{3}$ Institute of Health Sciences, VU University, De Boelelaan 1085, 1081 HV Amsterdam, The Netherlands \\ ${ }^{4}$ Department of Paediatrics, EMGO Institute, Institute of Cardiovascular Research, VU University Medical Centre, \\ PO Box 7057, 1007 MB Amsterdam, The Netherlands \\ (Submitted 12 September 2011 - Final revision received 16 February 2012 - Accepted 3 March 2012 - First published online 4 May 2012)
}

\begin{abstract}
Infant nutrition may influence eating behaviour and food preferences in later life. The present study explores whether exclusive breastfeeding duration and age at introduction of solid foods are associated with children's eating behaviour and fruit and vegetable intake at age 5 years. Data were derived from the Amsterdam Born Children and their Development study, a prospective birth cohort in the Netherlands, and included 3624 children. During infancy, data on infant nutrition were collected. Child eating behaviour (satiety responsiveness, enjoyment of food, slowness in eating and food responsiveness) was assessed with the Children's Eating Behaviour Questionnaire; and fruit and vegetable intake was calculated from a validated child FFQ. Both questionnaires were filled in by the mothers after their child turned 5 years. Exclusive breast-feeding duration was not associated with later eating behaviour, although longer exclusive breast-feeding was significantly associated with a higher vegetable intake at age 5 years. Compared with the introduction of solid foods at age 6 months, introduction before the age of 4 months was associated with less satiety responsiveness at age 5 years $(\beta-0.09 ; 95 \%$ $\mathrm{CI}-0 \cdot 16,-0 \cdot 02)$. Introducing solid foods after 6 months was associated with less enjoyment of food $(\beta-0 \cdot 07 ; 95 \% \mathrm{CI}-0 \cdot 12,-0 \cdot 01)$ and food responsiveness $(\beta-0.04 ; 95 \% \mathrm{CI}-0.07,-0 \cdot 01)$. Introducing solid foods before the age of 4 months was associated with a higher fruit intake compared with introduction at 6 months. These findings suggest that prolonged breast-feeding and introduction of solid foods between 4 and 6 months may lead to healthier eating behaviour and food preferences at age 5 years.
\end{abstract}

\section{Key words: Infant nutrition: Eating behaviour: Food preferences: Children}

Unhealthy eating habits in later life, and related unfavourable body composition and metabolic diseases, may be determined by early nutritional factors. Numerous studies have suggested that breast-feeding has beneficial effects on growth ${ }^{(1,2)}$, body composition $^{(3-5)}$ and related metabolic functions ${ }^{(6-8)}$, although in some studies this positive effect was absent ${ }^{(9)}$. The mechanism responsible for the beneficial effect of breast-feeding is not yet fully understood.

There are indications that breastfed infants may learn to regulate their energy intake or recognise satiety signals more effectively $^{(10)}$. Breastfed infants can control the amount of milk they consume, whereas bottle-fed infants may be encouraged by their mothers to finish a bottle even if they are satiated.
This overnutrition may lead to reduced satiety responses, as demonstrated in a study in which bottle-fed infants were more likely to empty milk in a cup or bottle in late infancy than breastfed infants ${ }^{(11)}$. Animal studies also show convincing results with respect to this satiety hypothesis, as early postnatal overfeeding leads to an increased appetite and food consumption, which persists into adulthood ${ }^{(12,13)}$ and to increased weight gain and body fat, which can lead to overweight and metabolic alterations similar to those in the human metabolic syndrome ${ }^{(12,14)}$.

In concordance with the satiety hypothesis, mothers who breastfeed their infants for at least 6 months show less controlling feeding styles ${ }^{(15)}$. It is known that maternal feeding

Abbreviations: ABCD, Amsterdam Born Children and their Development; CEBQ, Children's Eating Behaviour Questionnaire. 
styles influence children's eating behaviour ${ }^{(16)}$ and therefore breast-feeding may also indirectly contribute to children's natural satiety recognition and thus to the development of overweight.

Besides children's eating behaviour, infant nutrition may also influence later diet preferences. If mothers regularly eat healthy food during breast-feeding, this leads to more acceptance of these foods in infants ${ }^{(17)}$, possibly because some flavours are present in breast milk. Breastfed infants, who are repeatedly exposed, may therefore respond better to these familiar flavours at a later age ${ }^{(18,19)}$. Recently, Burnier et al. ${ }^{(20)}$ found that children who were exclusively breastfed for 3 or more months consume more vegetables at age 4 years than other infants. In contrast, Cooke et al. ${ }^{(21)}$ found that fruit consumption was associated with duration of breast-feeding, whereas they did not find any such evidence for vegetable intake. Thus, the relationship between breast-feeding and food preferences remains unclear.

Delayed introduction of solid foods also seems to protect against childhood overweight and obesity ${ }^{(22,23)}$, although this finding has not always been replicated ${ }^{(24,25)}$. Whether this is due to altered eating behaviour in later life has, to our knowledge, not been studied in humans. However, evidence from animal studies has shown that early-weaned rats reach satiety in adulthood slower than animals that underwent later weaning $^{(26)}$. There is evidence that infants who were introduced to complementary foods before 4 months of age are more likely to consume unhealthier food at the age of 1 year, even after correction for socio-demographic characteristics ${ }^{(27)}$. Whether these preferences persist in later life is unknown. However, studies in rats show that early weaning promotes an increased preference for palatable and fatty foods in later life, indicating permanent effects ${ }^{(28)}$.

The present study is the first large prospective study to explore the association between infant nutrition (duration of exclusive breast-feeding and age at introduction of solid foods) and children's eating behaviour and fruit and vegetable intake at age 5 years. Data were derived from an extensive unselected cohort of mothers and their children in Amsterdam, which allows for the correction of important confounders such as socio-economic status and maternal $\mathrm{BMI}^{(3,4,20,22,29)}$. We hypothesised that a long duration of exclusive breast-feeding and late introduction of solid foods would be associated with healthy eating behaviour and a higher fruit and vegetable intake.

\section{Methods \\ Study population}

Data collection was part of the Amsterdam Born Children and their Development (ABCD) study. The ABCD study is a longitudinal community-based cohort study which examines the association between early-life factors and later-health and health differences. The design and rationale of the ABCD study have already been described ${ }^{(30)}$. In brief, between January 2003 and March 2004, 8266 pregnant women (living in Amsterdam) were included in the ABCD study after their first prenatal visit to an obstetric caregiver. They filled out an extensive questionnaire, which included questions about socio-demographic data, obstetric history, lifestyle, dietary habits and psychosocial factors (phase one).

Of these respondents, 7863 women gave birth to a viable singleton infant and 6575 gave permission to collect growth and infant feeding data of the child in the following years, obtained by the Youth Health Care registration, the Department of the Public Health Service Amsterdam that provides preventive care for children from birth until adulthood (multiple births excluded). Then, 3 months after delivery, these mothers received another questionnaire, the infancy questionnaire, concerning the course of their pregnancy and delivery, their physical and psychosocial health and the baby's health, feeding, growth and development. This questionnaire was returned by 5131 mothers (phase two).

Phase three of the study started in the summer of 2008. About 2 weeks after the children turned 5 years, 6161 mothers of singletons who initially gave permission for follow-up received a questionnaire and an informed consent sheet for the child's participation in the ABCD health check. This questionnaire contained items about family socio-demographics and history of medical conditions, maternal lifestyle and psychosocial conditions and child's health, development and behaviour. Furthermore, it contained the Children's Eating Behaviour Questionnaire (CEBQ). Then, 2 weeks before the health check, the mothers received a notifying letter and an additional self-administered validated FFQ for young children ${ }^{(31)}$. During the preparation of this article, data collection was still ongoing. The CEBQ was completed and digitalised for 4382 children; and likewise with the FFQ for 2501 children.

Data on eating behaviour and infant feeding were available for 3654 children. Exclusion criteria were gluten and cow's milk allergies ( $n$ 23) and congenital disorders $(n 7)$. The present study included 3624 singleton children. Fruit and vegetable intake, based on the FFQ, was calculated for 2247 and 2253 children, respectively. The present study was conducted according to the guidelines laid down in the Declaration of Helsinki, and all procedures involving human subjects were approved by the review boards of all Amsterdam hospitals and the Registration Committee of Amsterdam. Written informed consent was obtained from all subjects.

\section{Measurements}

Children's eating behaviour at age 5 years was assessed with the CEBQ, a questionnaire developed by Wardle et al. ${ }^{(32)}$ that has been validated in Dutch ${ }^{(33)}$. The CEBQ is a parent-report measure designed to assess variation in eating style in children. The CEBQ consists of eight scales, of which we used four: (1) enjoyment of food and (2) food responsiveness, both representing interest in food and responsiveness to environmental food cues, (3) satiety responsiveness, which reflects a response to internal satiety cues, and (4) slowness in eating, which is characterised by a reduction in eating rate because of lack of enjoyment and interest in food. High scores on enjoyment of food and food responsiveness, and low scores on satiety responsiveness and slowness in eating, are associated with overweight and obesity in children ${ }^{(34-36)}$. The four scales included 
eighteen items, with each item scored on a four-point scale ( 1 = definitely not true, $2=$ not true, $3=$ true, $4=$ definitely true). The average scores on the four scales were calculated, with a minimum average score of 1 and a maximum score of 4. Scores were only calculated if at least $70 \%$ of the items were completed. Internal reliabilities of the CEBQ scales were acceptable, with Cronbach's $\alpha$ ranging from 0.74 to $0 \cdot 80$. Fruit and vegetable intake in $\mathrm{g} / \mathrm{d}$ was assessed with an FFQ that has been validated in Dutch for children aged $4-6$ years ${ }^{(31)}$.

Information on duration of breast-feeding and introduction of formula feeding was available from the infancy questionnaire received when the child was 3 months old, and from the Youth Health Care registration. This prospectively collected information was combined with retrospective information of the 5-year questionnaire to complete the data $(19.9 \%$ for breast-feeding and $25.4 \%$ for formula feeding). The intra-class correlation coefficient, an index of the reliability between the sources, was 0.64 for formula feeding and 0.81 for breastfeeding $(P<0.05)$, indicating that breast-feeding and formula feeding can reliably be reported retrospectively after 5 years. Information on introduction of solid foods was obtained from the Youth Health Care registration and the 5-year questionnaire $(95.9 \%)$. Duration of exclusive breast-feeding was categorised into: none, $<1,1-2 \cdot 9,3-6$ and $\geq 6$ months; and was defined as the period in which children received breastfeeding and did not receive any formula feeding or solid foods. Age of the child when solid foods were introduced was categorised into: $<4,4-5,6$ and $>6$ months.

In the analyses, we corrected for maternal factors: age (years), pre-pregnancy BMI $\left(\mathrm{kg} / \mathrm{m}^{2}\right)$ based on self-reported height and weight, ethnicity (Dutch, Mediterranean, African, other) defined by country of birth of the mother and her mother and educational level (years of education after primary school; $\leq 5,6-10,>10$ years) and for child factors: birth weight $(\mathrm{g})$, gestational age (weeks) and sex. Maternal factors were obtained from the pregnancy questionnaire, and child factors were obtained from the Youth Health Care registration.

\section{Statistical analyses}

Differences in group characteristics as a function of feeding pattern at age 6 months were examined with the $\chi^{2}$ test (categorical data) or ANOVA (continuous data). The following groups were compared post hoc with the exclusive breast-feeding group: breast-feeding combined with formula feeding (and solid foods), formula feeding and/or solid foods, and breast-feeding combined with solid foods. For each of the CEBQ scales and for fruit and vegetable intake, associations with each of the independent variables (duration of exclusive breast-feeding and introduction of solid foods) were explored using linear regression analyses. First, a crude model was analysed, with only the independent variables added to the model. In a second model, all potential confounders (maternal pre-pregnancy BMI, age, education and ethnicity and child birth weight, gestational age and sex) were added.

Significance was accepted at $P<0 \cdot 05$. Food responsiveness, a CEBQ subscale, was log-transformed to better resemble a normal distribution, as it was positively skewed. All statistical analyses were performed with SPSS version 18.0 (SPSS, Inc.).

\section{Results}

The group that was initially approached for phase three of the study, but did not respond ( $n$ 2518), and the group that was used for these analyses ( $n$ 3624) were similar with respect to sex $(50.6 v$ v. $49.9 \%$ girls). The participating mothers were older, had a lower pre-pregnancy BMI, a higher education and were more often of Dutch origin compared with the nonresponders $(P<0 \cdot 001)$. Children of participating mothers had a higher birth weight $(P<0 \cdot 001)$ (data not shown).

Table 1 presents the group characteristics, stratified for the feeding pattern of the child at 6 months of age. A total of 467 children (13\%) were fed according to the WHO guidelines ${ }^{(37)}$, which recommend exclusive breast-feeding for 6 months. Fruit and vegetable intake at age 5 years was, on average, 137 (SD 70) and 79 (SD 50) g/d, respectively. Mothers who exclusively breastfed for at least 6 months were generally older, and less often of African and more often of Mediterranean origin compared with mothers who did not breastfeed their child at age 6 months $(P<0 \cdot 05)$. Their children had a higher birth weight, on average. Furthermore, exclusively breastfed children had a higher vegetable intake at age 5 years than children who were not breastfed ( $P=0 \cdot 004)$. Mothers who combined breast-feeding with solid foods when their child was 6 months old had a higher pre-pregnancy BMI than mothers who exclusively breastfed $(P<0 \cdot 05)$. There were no differences in mean CEBQ scores between the groups.

\section{Exclusive breast-feeding}

In the crude analyses, children who were exclusively breastfed for 3-6 months scored higher on food responsiveness than children who received exclusive breast-feeding for at least 6 months (Table 2). In comparison with children who were exclusively breastfed for 6 months or longer, a significantly lower vegetable intake was found for children who were never exclusively breastfed, or exclusively breastfed for 1-2.9 months. No associations were found with fruit intake.

After adjustment for maternal and child factors, only children who were never exclusively breastfed had a lower vegetable intake than children who were exclusively breastfed for 6 months or longer. Also, children who were exclusively breastfed for 3-6 months still scored higher on food responsiveness than children who received exclusive breast-feeding for at least 6 months.

\section{Solid foods}

In the crude analyses, children who received solid foods before the age of 4 months had lower scores on satiety responsiveness than children who received solid foods at age 6 months (Table 3). Children who were introduced to solid foods after the age of 6 months had lower scores on enjoyment of food and food responsiveness than children who were introduced 
Table 1. Child and maternal characteristics by child's nutritional status at age 6 months

(Mean values and standard deviations)

\begin{tabular}{|c|c|c|c|c|c|c|c|c|c|}
\hline & \multicolumn{2}{|c|}{$\begin{array}{l}\text { Exclusive } \\
\text { breast-feeding } \\
\quad(n 467)\end{array}$} & \multicolumn{2}{|c|}{$\begin{array}{l}\text { Breast-feeding and } \\
\text { solid foods ( } n \text { 130) }\end{array}$} & \multicolumn{2}{|c|}{$\begin{array}{l}\text { Breast-feeding and } \\
\text { formula feeding } \\
\text { (and solid foods) } \\
\quad(n 676)\end{array}$} & \multicolumn{2}{|c|}{$\begin{array}{l}\text { Formula feeding/ } \\
\text { solid foods ( } n \text { 2351) }\end{array}$} & \multirow[b]{2}{*}{$P$} \\
\hline & Mean & SD & Mean & SD & Mean & SD & Mean & SD & \\
\hline \multicolumn{10}{|l|}{ Maternal factors } \\
\hline Pre-pregnancy BMI $\left(\mathrm{kg} / \mathrm{m}^{2}\right)$ & $22 \cdot 8$ & 3.5 & $23 \cdot 8^{\star}$ & $5 \cdot 2$ & $22 \cdot 6$ & 3.4 & $22 \cdot 9$ & 3.8 & 0.009 \\
\hline$\%$ Overweight & \multirow{2}{*}{\multicolumn{2}{|c|}{$\begin{array}{c}18.0 \\
4.1\end{array}$}} & \multirow{2}{*}{\multicolumn{2}{|c|}{$\begin{array}{c}19.2 \\
6.9\end{array}$}} & \multirow{2}{*}{\multicolumn{2}{|c|}{$\begin{array}{c}12 \cdot 9 \\
4.3\end{array}$}} & \multirow{2}{*}{\multicolumn{2}{|c|}{$\begin{array}{c}15.5 \\
5.1\end{array}$}} & \\
\hline$\%$ Obesity & & & & & & & & & \\
\hline Age (years) & $32 \cdot 2$ & 4.7 & 31.9 & 4.3 & $32 \cdot 3$ & 4.5 & $31 \cdot 7$ & $4 \cdot 6$ & 0.006 \\
\hline Education (\%) & & & & & & & \multirow{2}{*}{\multicolumn{2}{|c|}{$14 \cdot 7$}} & 0.020 \\
\hline $0-5$ years & \multicolumn{2}{|c|}{$14 \cdot 0$} & \multicolumn{2}{|c|}{$6 \cdot 2$} & \multicolumn{2}{|c|}{$13 \cdot 8$} & & & \\
\hline $6-10$ years & \multicolumn{2}{|c|}{$32 \cdot 8$} & \multicolumn{2}{|c|}{$\begin{array}{l}34.6 \\
59.2\end{array}$} & \multicolumn{2}{|c|}{$33 \cdot 2$} & \multicolumn{2}{|c|}{$36 \cdot 9$} & \\
\hline$>10$ years & \multicolumn{2}{|c|}{$53 \cdot 1$} & \multicolumn{2}{|c|}{$59 \cdot 2$} & \multicolumn{2}{|c|}{53.0} & \multicolumn{2}{|c|}{$48 \cdot 4$} & \\
\hline Ethnicity (\%) & & & & & & & & & $0.003 \dagger$ \\
\hline Dutch & \multicolumn{2}{|c|}{$64 \cdot 6$} & \multicolumn{2}{|c|}{$66 \cdot 2$} & \multicolumn{2}{|c|}{59.5} & \multicolumn{2}{|c|}{$65 \cdot 5$} & \\
\hline African & \multicolumn{2}{|c|}{$2 \cdot 6$} & \multicolumn{2}{|c|}{$3 \cdot 8$} & \multicolumn{2}{|c|}{$4 \cdot 3$} & \multicolumn{2}{|c|}{$5 \cdot 7$} & \\
\hline Mediterranean & & & & & & & & & \\
\hline Other & & & & & & & & & \\
\hline Child factors & & & & & & & & & \\
\hline $\operatorname{Sex}(\%)$ & & & & & & & & & 0.826 \\
\hline Girl & & & & & & & & & \\
\hline Boy & & & & & & & & & \\
\hline Birth weight (g) & 3549.0 & $502 \cdot 0$ & $3631 \cdot 0$ & $497 \cdot 0$ & 3499.0 & $498 \cdot 0$ & $3449.0^{*}$ & $559 \cdot 0$ & $<0.001$ \\
\hline Gestational age (weeks) & $40 \cdot 0$ & 1.5 & $40 \cdot 1$ & 1.4 & $40 \cdot 0$ & 1.6 & 39.8 & 1.7 & 0.002 \\
\hline Enjoyment of food & 2.53 & 0.47 & 2.47 & 0.47 & 2.53 & 0.51 & 2.53 & 0.50 & 0.645 \\
\hline Satiety responsiveness & $2 \cdot 36$ & 0.48 & 2.38 & 0.58 & $2 \cdot 35$ & 0.52 & $2 \cdot 37$ & 0.49 & 0.711 \\
\hline Slowness in eating & $2 \cdot 41$ & 0.58 & 2.51 & 0.59 & 2.46 & 0.57 & $2 \cdot 47$ & 0.57 & 0.194 \\
\hline Food responsiveness & 1.83 & 0.47 & 1.86 & 0.53 & 1.89 & 0.50 & 1.90 & 0.52 & 0.092 \\
\hline Fruit intake $(\mathrm{g} / \mathrm{d}) \ddagger$ & $141 \cdot 2$ & $73 \cdot 6$ & $149 \cdot 6$ & $70 \cdot 8$ & $143 \cdot 1$ & $67 \cdot 3$ & $133 \cdot 8$ & $69 \cdot 8$ & 0.019 \\
\hline Vegetable intake $(\mathrm{g} / \mathrm{d}) \ddagger$ & $85 \cdot 6$ & $49 \cdot 7$ & $87 \cdot 8$ & $54 \cdot 2$ & $81 \cdot 3$ & $51 \cdot 8$ & $76 \cdot 1^{\star}$ & $49 \cdot 3$ & 0.004 \\
\hline
\end{tabular}

${ }^{*}$ Mean values are significantly different from the exclusive breast-feeding group.

$\dagger$ Ethnic composition in the formula feeding/solid foods group is significantly different from the exclusive breast-feeding group.

$\ddagger$ Based on a subsample, $n 2247$ for fruit intake and $n 2253$ for vegetable intake.

to solid foods at age 6 months. After adjusting for confounders,

the differences in eating behaviour remained significant.

Children who received solid foods before 4 months of age had a higher fruit intake than children who received solid foods at age 6 months; this difference became significant after adjustment for confounders. No associations were found with vegetable intake.

As there was a correlation between the age at which solid foods were introduced and other infant nutrition, we also corrected for duration of breast-feeding and introduction of formula feeding besides all covariates. However, this had no effect on the observed associations.

\section{Discussion}

The present study indicates that the age at which solid foods are introduced may influence later eating behaviour. We found that children who were introduced to solid foods before the age of 4 months were less able to recognise satiety signals at the age of 5 years, whereas a late introduction to solid foods ( $>6$ months) was associated with less food enjoyment and food responsiveness. Vegetable intake, on the other hand, seemed to be more related to breast-feeding and formula feeding. Breast-feeding exclusively for a longer period of time and thus delaying formula feeding was associated with a higher vegetable intake at age 5 years.
To our knowledge, this is the first study to examine infant feeding in relation to eating behaviour, measured with the CEBQ. It has been shown that breast-feeding protects against later overweight and obesity ${ }^{(3-5)}$. Besides the hormonal influences of leptin and adiponectin in breastmilk ${ }^{(38-41)}$, this protection may be partly due to the ability of breastfed infants to regulate their energy intake in contrast to formula-fed infants, as discussed by Li et al. ${ }^{(11)}$. However, we found no association between the duration of exclusive breast-feeding and later eating behaviour, except for a higher score on food responsiveness for children who were exclusively breastfed for 1-2.9 months. This latter finding is probably more by chance, as no linear trend in food responsiveness in relation to breast-feeding or formula feeding was seen. The effect of breast-feeding on satiety recognition and other eating behaviour may only be present during infancy. It is also possible that the feeding style, and not the type of milk, affects the self-regulation of infants, as Li et al. ${ }^{(11)}$ found that directly breastfed infants are more able to control their intake in comparison with both formula-fed infants and infants who were fed expressed human milk by bottle. Unfortunately, in the present study, expressed milk and milk fed directly at the breast could not be examined separately. Future research should assess the differences in eating behaviour between directly breastfed infants and infants fed expressed milk by bottle. 
Besides a short duration of breast-feeding, early introduction of solid foods (before the age of 4 months) may also increase the risk of later overweight ${ }^{(22,23)}$. This may be partly explained by eating behaviour, as the present study shows that these children are less capable to recognise satiety signals.

Although the age at introduction of solid foods may programme later eating behaviour, it is also possible that children with bigger appetites (compared with other children) receive complementary foods at a young age, because their mothers perceive them as being hungry. However, the fact that animal studies have shown that early weaning can influence satiety behaviour supports the programming model ${ }^{(26)}$. Children's eating behaviour may be influenced by parental eating behaviour, as children of obese parents show different eating behaviour compared with children of lean families ${ }^{(42)}$. Since we lack information on parental eating behaviour, this may have influenced the relationship between the introduction of solid foods and child eating behaviour.

Prenatal as well as infant nutrition may influence later food preferences. For example, individuals prenatally exposed to the Dutch famine showed a preference for fatty foods later in life ${ }^{(43)}$. Burnier et al. ${ }^{(20)}$ found that children who were exclusively breastfed for 3 or more months consume more vegetables at age 4 years than other infants. In concordance with this, we found the lowest vegetable intake at age 5 years in children who were never exclusively breastfed and were thus introduced to formula feeding at birth. The duration of exclusive breastfeeding showed a linear association with fruit and vegetable intake, although not always significant. Exclusive breastfeeding for a longer period of time was associated with an increasing intake of both fruit and vegetables. These associations may be due to the fact that breast-feeding promotes healthier food preferences, as some flavours of healthy foods may be present in breast milk, and breastfed infants recognise these flavours and therefore respond better to them ${ }^{(18,19)}$. However, mothers who breastfeed for 6 months or longer may also have a healthier lifestyle and offer their child more fruits and vegetables, as they are more conscientious about their child's health. Although we corrected for socio-economic status and BMI, which are strongly related to lifestyle, some residual confounding is still possible. The reason why no significant differences were found in fruit intake may be that fruit is the most common first food for infants in the Netherlands and therefore all children have a high fruit intake. Significant differences are then more difficult to detect.

In addition to breast-feeding, the introduction of solid foods may also influence food preferences. Earlier observational and experimental studies found an unhealthy and fattier food preference when complementary foods were introduced at a young age ${ }^{(27,28)}$. In contrast with these studies, we found a higher fruit intake in children who were introduced to solid foods before the age of 4 months. This may be because these children have a big appetite and like food in general or because they have eaten more fruit at a young age (fruit is the most common first food for infants in the Netherlands) and have therefore developed a preference for fruit.

The major strengths of this study were the prospective design and the large study population. Also, we were able to control for 
several confounding factors. Nevertheless, a few limitations should be discussed. First, we lacked information on maternal diet. Parental fruit and vegetable intake is a predictor of children's fruit and vegetable intake ${ }^{(21)}$. The parental diet is, however, related to socio-economic status and BMI, both of which we corrected for in our analyses. Therefore, we expect little residual confounding by maternal food preferences. Second, we were unable to correct for the children's total energy intake, as we did not measure the total dietary pattern of the children. This correction would ensure that the differences in fruit and vegetable intake and eating behaviour were not explained by a bigger appetite in general in some children. Furthermore, as the study population under-represents mothers with a low socio-economic status, the results may not be applicable to the entire Dutch population. However, this has probably led to an underestimation of effects, as mothers from low socio-economic groups introduce solid foods at an earlier age and are less likely to breastfeed ${ }^{(44-46)}$. Third, most of the information was collected prospectively; however, some data on infant nutrition were completed with retrospective information. This might lead to recall bias. However, for breast-feeding and formula feeding, the intra-class correlation coefficient was quite sufficient. Lastly, food responsiveness was positively skewed in the present study, which is not in accordance with other studies. It may be caused by our multi-ethnic population or young age group, as the children in this study were younger than in most other studies that use the CEBQ However, some smaller studies also used 5-year-old children and did not find skewness in food responsiveness ${ }^{(35,42)}$. The smaller number of these studies $(n<500)$ could explain the incongruent results.

The $\mathrm{WHO}^{(37)}$ recommendations state that children should be exclusively breastfed for 6 months. The present study shows that exclusive breast-feeding for a long period of time is associated with a higher fruit and vegetable intake. The introduction of solid foods before 4 months should be discouraged, as these children seem to be less able to recognise satiety signals. However, the introduction of complementary foods after 6 months is also not recommended, as this may lead to a lower fruit intake (as indicated in the present study), and is associated with nutrient deficiencies and the development of allergies $^{(47,48)}$. Although the differences in fruit and vegetable intake between the groups are small, they can have large health consequences, as average fruit $(137 \mathrm{~g} / \mathrm{d})$ and vegetable $(79 \mathrm{~g} / \mathrm{d})$ intakes were already below the Dutch guidelines for children aged $4-8$ years, which recommend $150 \mathrm{~g}$ of fruit and $100-150 \mathrm{~g}$ of vegetables per $\mathrm{d}^{(49)}$. Therefore, an even lower intake in some children may lead to nutrient deficiencies. Other studies also indicate that the introduction of complementary feeding between the ages of 4 and 6 months is the best choice ${ }^{(47,48)}$.

In conclusion, the present study shows that a long duration of breast-feeding and the introduction of solid foods between the ages of 4 and 6 months are associated with healthy eating behaviour and healthy food choices. 


\section{Acknowledgements}

The ABCD study was funded by The Netherlands Organisation for Health Research and Development (ZonMw). The authors declare that they have no competing interests. L. M. M. drafted the manuscript and conducted the analyses. T. G. M. V., M. v. E. and R. J. B. J. G. designed the study and obtained funding. All authors made substantial contributions to conception, design and revising the manuscript. All authors read and approved the final manuscript.

\section{References}

1. Dewey KG (1998) Growth characteristics of breast-fed compared to formula-fed infants. Biol Neonate $\mathbf{7 4}$, 94-105.

2. Kramer MS, Guo T, Platt RW, et al. (2004) Feeding effects on growth during infancy. J Pediatr 145, 600-605.

3. Arenz S, Ruckerl R, Koletzko B, et al. (2004) Breast-feeding and childhood obesity - a systematic review. Int $J$ Obes Relat Metab Disord 28, 1247-1256.

4. Owen CG, Martin RM, Whincup PH, et al. (2005) Effect of infant feeding on the risk of obesity across the life course: a quantitative review of published evidence. Pediatrics 115, 1367-1377.

5. Monasta L, Batty GD, Cattaneo A, et al. (2010) Early-life determinants of overweight and obesity: a review of systematic reviews. Obes Rev 11, 695-708.

6. Martin RM, Gunnell D \& Smith GD (2005) Breastfeeding in infancy and blood pressure in later life: systematic review and meta-analysis. Am J Epidemiol 161, 15-26.

7. Owen CG, Martin RM, Whincup PH, et al. (2006) Does breastfeeding influence risk of type 2 diabetes in later life? A quantitative analysis of published evidence. Am J Clin Nutr 84, 1043-1054.

8. Owen CG, Whincup PH, Kaye SJ, et al. (2008) Does initial breastfeeding lead to lower blood cholesterol in adult life? A quantitative review of the evidence. Am J Clin Nutr 88, 305-314.

9. Kramer MS (2010) 'Breast is best': the evidence. Early Hum Dev 86, 729-732.

10. Li R, Fein SB \& Grummer-Strawn LM (2008) Association of breastfeeding intensity and bottle-emptying behaviors at early infancy with infants' risk for excess weight at late infancy. Pediatrics 122, Suppl. 2, S77-S84.

11. Li R, Fein SB \& Grummer-Strawn LM (2010) Do infants fed from bottles lack self-regulation of milk intake compared with directly breastfed infants? Pediatrics 125, e1386-e1393.

12. Plagemann A, Harder T, Rake A, et al. (1999) Perinatal elevation of hypothalamic insulin, acquired malformation of hypothalamic galaninergic neurons, and syndrome $\mathrm{x}$-like alterations in adulthood of neonatally overfed rats. Brain Res 836, $146-155$.

13. Rodrigues AL, de Moura EG, Passos MC, et al. (2009) Postnatal early overnutrition changes the leptin signalling pathway in the hypothalamic-pituitary-thyroid axis of young and adult rats. J Physiol 587, 2647-2661.

14. Plagemann A, Harder T, Rake A, et al. (1999) Observations on the orexigenic hypothalamic neuropeptide Y-system in neonatally overfed weanling rats. I Neuroendocrinol 11, 541-546.

15. Taveras EM, Scanlon KS, Birch L, et al. (2004) Association of breastfeeding with maternal control of infant feeding at age 1 year. Pediatrics 114, e577-e583
16. Webber L, Cooke L, Hill C, et al. (2010) Associations between children's appetitive traits and maternal feeding practices. J Am Diet Assoc 110, 1718-1722.

17. Forestell CA \& Mennella JA (2007) Early determinants of fruit and vegetable acceptance. Pediatrics 120, 1247-1254.

18. Mennella JA (1995) Mother's milk: a medium for early flavor experiences. J Hum Lact 11, 39-45.

19. Mennella JA \& Beauchamp GK (1991) Maternal diet alters the sensory qualities of human milk and the nursling's behavior. Pediatrics 88, 737-744.

20. Burnier D, Dubois L \& Girard M (2011) Exclusive breastfeeding duration and later intake of vegetables in preschool children. Eur J Clin Nutr 65, 196-202.

21. Cooke LJ, Wardle J, Gibson EL, et al. (2004) Demographic, familial and trait predictors of fruit and vegetable consumption by pre-school children. Public Health Nutr 7, 295-302.

22. Seach KA, Dharmage SC, Lowe AJ, et al. (2010) Delayed introduction of solid feeding reduces child overweight and obesity at 10 years. Int J Obes (Lond) 34, 1475-1479.

23. Wilson AC, Forsyth JS, Greene SA, et al. (1998) Relation of infant diet to childhood health: seven year follow up of cohort of children in Dundee infant feeding study. BMJ 316, 21-25.

24. Burdette HL, Whitaker RC, Hall WC, et al. (2006) Breastfeeding, introduction of complementary foods, and adiposity at $5 \mathrm{y}$ of age. Am J Clin Nutr 83, 550-558.

25. Reilly JJ, Armstrong J, Dorosty AR, et al. (2005) Early life risk factors for obesity in childhood: cohort study. BMJ 330, 1357.

26. dos Santos Oliveira L, da Silva LP, da Silva AI, et al. (2011) Effects of early weaning on the circadian rhythm and behavioral satiety sequence in rats. Behav Process 86, 119-124.

27. Grummer-Strawn LM, Scanlon KS \& Fein SB (2008) Infant feeding and feeding transitions during the first year of life. Pediatrics 122, Suppl. 2, S36-S42.

28. dos Santos Oliveira L, de Lima DP, da Silva AA, et al. (2011) Early weaning programs rats to have a dietary preference for fat and palatable foods in adulthood. Behav Process 86, 75-80.

29. Huh SY, Rifas-Shiman SL, Taveras EM, et al. (2011) Timing of solid food introduction and risk of obesity in preschool-aged children. Pediatrics 127, e544-e551.

30. Van Eijsden M, Vrijkotte TG, Gemke RJ, et al. (2011) Cohort profile: The Amsterdam Born Children and their Development (ABCD) Study. Int J Epidemiol 40, 1176-1186.

31. Dutman AE, Stafleu A, Kruizinga A, et al. (2010) Validation of an FFQ and options for data processing using the doubly labelled water method in children. Public Health Nutr 14, 410-417.

32. Wardle J, Guthrie CA, Sanderson S, et al. (2001) Development of the Children's Eating Behaviour Questionnaire. J Child Psychol Psychiat 42, 963-970.

33. Sleddens EF, Kremers SP \& Thijs C (2008) The children's eating behaviour questionnaire: factorial validity and association with body mass index in Dutch children aged 6-7. Int J Behav Nutr Phys Act 5, 49.

34. Webber L, Hill C, Saxton J, et al. (2009) Eating behaviour and weight in children. Int J Obes (Lond) 33, 21-28.

35. Parkinson KN, Drewett RF, Le Couteur AS, et al. (2010) Do maternal ratings of appetite in infants predict later Child Eating Behaviour Questionnaire scores and body mass index? Appetite 54, 186-190.

36. Carnell S \& Wardle J (2008) Appetite and adiposity in children: evidence for a behavioral susceptibility theory of obesity. Am J Clin Nutr 88, 22-29. 
37. World Health Organization (2001) The Optimal Duration of Exclusive Breastfeeding: Report of an Expert Consultation. Geneva: World Health Organization.

38. Houseknecht KL, McGuire MK, Portocarrero CP, et al. (1997) Leptin is present in human milk and is related to maternal plasma leptin concentration and adiposity. Biochem Biophys Res Commun 240, 742-747.

39. Qi Y, Takahashi N, Hileman SM, et al. (2004) Adiponectin acts in the brain to decrease body weight. Nat Med 10, 524-529.

40. Singhal A, Farooqi IS, O'Rahilly S, et al. (2002) Early nutrition and leptin concentrations in later life. Am J Clin Nutr 75, 993-999.

41. Newburg DS, Woo JG \& Morrow AL (2010) Characteristics and potential functions of human milk adiponectin. $J$ Pediatr 156, S41-S46.

42. Wardle J, Guthrie C, Sanderson S, et al. (2001) Food and activity preferences in children of lean and obese parents. Int J Obes Relat Metab Disord 25, 971-977.

43. Lussana F, Painter RC, Ocke MC, et al. (2008) Prenatal exposure to the Dutch famine is associated with a preference for fatty foods and a more atherogenic lipid profile. $\mathrm{Am} \mathrm{J}$ Clin Nutr 88, 1648-1652.
44. Wijndaele K, Lakshman R, Landsbaugh JR, et al. (2009) Determinants of early weaning and use of unmodified cow's milk in infants: a systematic review. J Am Diet Assoc 109, 2017-2028.

45. Li R, Darling N, Maurice E, et al. (2005) Breastfeeding rates in the United States by characteristics of the child, mother, or family: the 2002 National Immunization Survey. Pediatrics 115, e31-e 37

46. Ladomenou F, Kafatos A \& Galanakis E (2007) Risk factors related to intention to breastfeed, early weaning and suboptimal duration of breastfeeding. Acta Paediatr 96, 1441-1444.

47. Agostoni C, Decsi T, Fewtrell M, et al. (2008) Complementary feeding: a commentary by the ESPGHAN Committee on Nutrition. J Pediatr Gastroenterol Nutr 46, 99-110.

48. Anderson J, Malley K \& Snell R (2009) Is 6 months still the best for exclusive breastfeeding and introduction of solids? A literature review with consideration to the risk of the development of allergies. Breastfeed Rev 17, 23-31.

49. Voedingscentrum (2008) Gezond eten en bewegen met kinderen van 4-8 jaar (Healthy Eating and Physical Activity in Children Aged 4-8 Years). The Hague: Voedingscentrum. 